\title{
Caracterização físico-química de amostras de mel de abelha africanizada dos municípios de Santa Helena e Terra Roxa (PR)
}

\author{
[Physicochemical parameters of honey from samples from africanized honeybees in Santa Helena \\ and terra roxa counties $(P R)]$ \\ F.J. Moraes, R.C. Garcia, E. Vasconcelos, S.C. Camargo, B.G. Pires, A.M. Hartleben, \\ F. Liesenfeld, D.J. Pereira, E.S. Mittanck, J. Giasson, J.R. Gremaschi \\ Universidade Estadual do Oeste do Paraná (UNIOESTE) - Foz do Iguaçu, PR
}

\begin{abstract}
RESUMO
O presente estudo teve como objetivo a caracterização físico-química de amostras de mel de Apis mellifera coletadas nos municípios de Santa Helena e Terra Roxa, localizados na região oeste do Estado do Paraná, Brasil. Foram coletadas 40 amostras de mel, sendo 20 do município de Santa Helena e 20 de Terra Roxa (PR), coletadas diretamente com os apicultores, as quais foram submetidas a análises físicoquímicas de umidade, acidez, $\mathrm{pH}$, cinzas, condutividade elétrica e cor, a fim de verificar se as mesmas apresentavam-se em conformidade com a legislação nacional vigente (Instrução Normativa $\mathrm{n}^{\circ} 11$ ), além de conhecer o perfil físico-químico do mel das diferentes localidades, na safra 2008/2009. Os dados encontrados foram submetidos à análise de variância e teste $\mathrm{F}$ a $5 \%$ de significância. Os resultados apresentaram diferença significativa $(\mathrm{P}<0,05)$ apenas entre os valores de acidez, tendo o mel oriundo de Terra Roxa apresentado valor mais alto $\left(33,45 \pm 7,7\right.$ meq. $\left.\mathrm{kg}^{-1}\right)$ que o de Santa Helena $\left(24,53 \pm 6,3 \mathrm{meq} \cdot \mathrm{kg}^{-1}\right)$. Em sua maioria, as amostras analisadas encontraram-se dentro das especificações determinadas pela legislação para as características físico-químicas, com exceção do parâmetro de umidade, que, apesar de não ter apresentado diferença significativa entre os valores encontrados para os dois municípios, estavam acima do limite estabelecido pela legislação (20\%) em 8 amostras do município de Santa Helena e em 7 de Terra Roxa, totalizando 37,5\% das amostras. Essa característica pode ter deixado o produto mais susceptível à fermentação e pode ter sido ocasionada pela colheita imatura do mel.
\end{abstract}

Palavras-chave: Apis mellifera, apicultura, composição do mel

\begin{abstract}
This study aimed to physicochemically characterize the honey samples of Apis mellifera collected in Santa Helena and Terra Roxa counties, located in the western region of Paraná State, Brazil. Forty honey samples were collected, 20 in Santa Helena and 20 in Terra Roxa (PR) counties, directly from beekeepers, and were subjected to physico-chemical analyzes of moisture, acidity, $\mathrm{pH}$, ashes, electrical conductivity and color, to verify whether they were in accordance with the existing national legislation (Instruction No. 11), in addition to knowing the physicochemical profile of different sites during the 2008/2009 season. The data were submitted to analysis of variance and $F$ test at 5\% significance level. The results showed significant differences $(p<0.05)$ between values of acidity, and the honey samples from Terra Roxa presented a higher value (33.45 \pm 7.7 meq. $\mathrm{kg}$-1) than Santa Helena $(24=53 \pm 6.3 \mathrm{meq} . \mathrm{kg}$ 1). Most of the analyzed samples were within the specifications prescribed in the legislation for the physico-chemical parameters except for moisture, which despite having presented a significant difference between values obtained from two counties, were above the limits established by the legislation (20\%) in eight samples in Santa Helena and seven in Terra Roxa counties, totaling 37.5\%. This characteristic may have made the product more susceptible to fermentation, and may have been caused by harvesting immature honey.
\end{abstract}

Keywords: beekeeping, Apis mellifera, honey composition

Recebido em 16 de maio de 2013

Aceito em 17 de dezembro de 2013

E-mail: fernanda_colt@msm.com 


\section{INTRODUÇÃO}

Devido ao crescimento da população mundial das últimas décadas, tornou-se necessário aumento na produção de alimentos frente à procura, por parte de consumidores, por produtos seguros e de qualidade. Como reflexo, a apicultura, que era feita de forma artesanal, passou a ser tecnificada, visando melhorar a quantidade e a qualidade do mel produzido.

A qualidade do mel depende de vários fatores, tais como: origem botânica do néctar coletado, espécie da abelha, condições ambientais e manejo pré e pós-colheita (Fujii et al., 2009). Diante da grande variabilidade em termos de composição do produto, o Ministério da Agricultura, Pecuária e Abastecimento instituiu em 20 de outubro de 2000 uma normativa específica, denominada Instrução Normativa $\mathrm{n}^{\circ}$ 11, que estabelece parâmetros de controle de qualidade do mel, com indicação das análises e métodos a serem empregados (Brasil, 2000).

Atualmente a produção melífera brasileira encontra-se principalmente na região sul, sendo o Rio Grande do Sul o principal produtor no ano de 2010, com o total de 7.090 toneladas de mel, e o Paraná, o segundo maior produtor, com 5.468 toneladas. O oeste desse Estado é responsável por 907.210 quilos, e os municípios de Santa Helena e Terra Roxa participam com 80.380 e 8.500 quilos, respectivamente (IBGE, 2010).

As características físico-químicas do mel paranaense são pouco conhecidas, principalmente na região oeste, onde a flora apícola é bastante diversificada devido a programas de reflorestamento em áreas de preservação permanente próximas ao lago de Itaipu, tornando-se de relevada importância um estudo abrangendo essa região.

Este trabalho teve como objetivo a caracterização físico-química (umidade, $\mathrm{pH}$, acidez, condutividade elétrica, cinzas e cor) de 40 amostras de mel de Apis mellifera provenientes dos municípios de Santa Helena, à margem do Lago de Itaipu, e Terra Roxa, ambos localizados na região oeste do Estado do Paraná.

\section{MATERIAL E MÉTODOS}

O presente estudo foi realizado no período de agosto de 2010 a dezembro de 2011, no Laboratório de Análise de Alimentos do Centro de Ciências Agrárias da Universidade Estadual do Oeste do Paraná - UNIOESTE, campus Marechal Cândido Rondon - PR.

Foram coletadas 40 amostras de mel de $A$. mellifera, fornecidas por apicultores associados à COOFAMEL - Cooperativa Agrofamiliar Solidária dos Apicultores da Costa Oeste do Paraná, provenientes da safra de 2008/2009, sendo 20 de produtores localizados nos municípios de Santa Helena e 20 de Terra Roxa, oeste do Estado do Paraná.

As amostras foram armazenadas em embalagens plásticas transparentes, com tampa de rosca, capacidade para 500g, especiais para armazenamento de produtos alimentícios, e encaminhadas ao laboratório, onde ficaram armazenadas pelo período de aproximadamente um ano, sem controle de temperatura e umidade, para posterior análise.

O município de Santa Helena fica localizado no oeste do Estado do Paraná, às margens do Lago de Itaipu (bacia hidrográfica do Paraná), com altitude de 258 metros, latitude $24^{\circ} 51^{\prime} 37^{\prime}$ 'S, Longitude $54^{\circ} 19^{\prime} 58^{\prime \prime}$ W-GR e área total de $754,7 \mathrm{~km}^{2}$. O clima é classificado como Cfa, segundo Köppen (1948), Subtropical Úmido Mesotérmico, verões quentes com tendência de concentração de chuvas (temperatura média superior a $22^{\circ} \mathrm{C}$ ), inverno com geadas pouco frequentes (temperatura média inferior a $18^{\circ} \mathrm{C}$ ), sem estação seca definida.

O município de Terra Roxa fica localizado no oeste do Paraná, com altitude de 260 metros, Latitude $24^{\circ} 09^{\prime} 40^{\prime \prime} \mathrm{S}$, Longitude $54^{\circ} 06^{\prime} 30^{\prime \prime} \mathrm{W}$ GR e área total de $845,38 \mathrm{~km}^{2}$.

O tipo climático predominante é o Cfa, segundo a classificação de Köppen (1948), subtropical úmido mesotérmico que se caracteriza pela predominância de verões quentes e invernos com baixa frequência de geadas severas, com tendência de concentração das chuvas nos meses 
de verão. A temperatura média anual é de aproximadamente $22^{\circ} \mathrm{C}$, concentrando-se os valores médios mensais mais elevados nos meses de janeiro e fevereiro, em torno de $28^{\circ} \mathrm{C}$, e períodos mais frios nos meses de junho, julho e agosto, com temperaturas médias mínimas mensais em torno de $18^{\circ} \mathrm{C}$.

As amostras foram colocadas em embalagens plásticas transparentes, com tampa de rosca, capacidade para 500g, especiais para armazenamento de produtos alimentícios, e encaminhadas ao laboratório, onde ficaram armazenadas pelo período de aproximadamente um ano, à temperatura ambiente, para posterior análise.

Para a caracterização do mel da região, foram efetuadas as seguintes análises físico-químicas: umidade, realizada pelo método descrito por
Association... (1990); cinzas, de acordo com o método adotado pelo Instituto Adolfo Lutz, descrito por Zenebon et al. (2008); pH e de acidez, pelo método proposto por Moraes e Teixeira (1998); condutividade elétrica, por Boletim... (1986); e cor, pelo método proposto por Vidal e Fregosi (1984).

Os dados encontrados na avaliação das características físico-químicas das amostras de mel dos municípios de Santa Helena e Terra Roxa foram submetidos à análise de variância convencional (ANOVA - one way) e teste $\mathrm{F}$ ao nível de $5 \%$ de significância.

\section{RESULTADOS E DISCUSSÃO}

Os resultados obtidos para as análises físicoquímicas das 40 amostras de mel de abelha $A$. mellifera estão representados na Tab. 1.

Tabela 1. Valores médios dos parâmetros determinados nas amostras de mel de Santa Helena e Terra Roxa, Estado do Paraná, produzido por Apis melífera, safra 2008/2009

\begin{tabular}{|c|c|c|c|c|c|c|}
\hline \multirow[b]{2}{*}{ Municípios } & \multirow[b]{2}{*}{$\mathrm{n}$} & \multicolumn{5}{|c|}{ Parâmetros físico-químicos } \\
\hline & & Umidade $(\%)$ & $\mathrm{pH}$ & $\begin{array}{l}\text { Acidez } \\
\left(\text { meq.kg }{ }^{-1}\right)\end{array}$ & $\begin{array}{l}\text { Condutividade } \\
\left(\mu \mathrm{S} . \mathrm{cm}^{-1}\right)\end{array}$ & $\begin{array}{l}\text { Cinzas } \\
(\%)\end{array}$ \\
\hline Terra Roxa & 20 & $19,22 \pm 1,4$ & $3,94 \pm 0,6$ & $33,45 \pm 7,7 *$ & $371,22 \pm 131,3$ & $0,19 \pm 0,1$ \\
\hline Santa Helena & 20 & $19,53 \pm 1,5$ & $3,98 \pm 0,1$ & $24,53 \pm 6,3$ & $391,83 \pm 50,5$ & $0,19 \pm 0,1$ \\
\hline P-valor & & 0,27 & 0,64 & 0,00 & 0,26 & 0,82 \\
\hline
\end{tabular}

*médias diferem estatisticamente entre si, na mesma coluna, pelo teste de $\mathrm{F}(\mathrm{P}<0,05)$.

Os teores de umidade encontrados nas amostras de mel não diferiram estatisticamente entre si ( $\mathrm{P}>0,05)$, obtendo médias de 19,22 $\pm 1,4$ e $19,53 \pm 1,5 \%$ para os municípios de Terra Roxa e Santa Helena, respectivamente, situando-se dentro dos parâmetros estabelecidos pela norma vigente no Brasil, que preconiza o máximo de $20 \%$ de umidade no mel. Observou-se ainda que, das 40 amostras de mel analisadas para o parâmetro umidade, $15 \quad(37,5 \%)$ delas encontram-se em desacordo com a legislação, sendo oito advindas do município de Santa Helena e sete de Terra Roxa.

No Estado do Paraná, Alves et al. (2011), estudando mel orgânico produzido em ilhas do Rio Paraná, encontraram valor médio de $18,85 \pm 0,18 \%$ para 24 amostras de mel, e Camargo (2011), estudando mel de duas áreas apícolas do município de Santa Helena (PR), encontrou os valores de 17,09 $\pm 1,05$ e $16,99 \pm 1,37$.

Segundo Marchini et al. (2004b), a água constitui o segundo componente do mel em termos de quantidade, geralmente variando de 15 a $21 \%$, dependendo do clima, origem floral e colheita prematura. Silva et al. (2004) citam que é provável que mel produzido durante a época chuvosa apresente maior umidade em função da saturação do ar e do grande fluxo de néctar mais diluído que ocorre logo após as chuvas, e que teores altos de umidade deixam o mel mais susceptível ao crescimento de microrganismos indesejáveis.

$\mathrm{O}$ alto número de amostras encontradas com o teor de umidade acima de $20 \%$ poderia ser explicado devido à colheita prematura do mel, ou à absorção de água do ambiente durante o armazenamento na propriedade, por ser 
altamente higroscópico, ou ainda pela quantidade de chuvas na época em que foi produzido, o que pode diminuir a sua vida de prateleira. Este fator pode ser indicativo do problema de manejo incorreto na região, semelhante ao que se observa em outras regiões do país.

Os valores médios de $\mathrm{pH}$ encontrados nas amostras de mel no município de Terra Roxa $(3,94 \pm 0,6)$ e Santa Helena $(3,98 \pm 01)$ não diferiram estatisticamente entre si, situando-se próximos às médias encontradas por Silva et al. (2004), em mel produzido no Estado do Piauí, de diferentes floradas, que variaram entre 3,54 a 5,30. Moreti et al. (2009) estudaram amostras de mel de A. mellifera provenientes do Estado do Ceará e encontraram valores médios de 3,6, variando entre 3,4 e 5,3, assim como Camargo (2011) que, também trabalhando com amostras de mel de duas áreas de Santa Helena (PR), encontrou $\mathrm{pH} 4,21 \pm 0,13$ e 4,26 $\pm 0,11$ para cada uma delas.

Conforme descrito por Silva et al. (2004), embora a análise de $\mathrm{pH}$ não seja indicada, atualmente, como análise obrigatória no controle de qualidade do mel brasileiro, esta mostra-se útil como variável auxiliar para avaliação da qualidade do mel, pois influencia na velocidade de formação do hidroximetilfurfural. Segundo Crane (1983), variações observadas no pH do mel se devem, provavelmente, a particularidades na composição florística das áreas de coleta, uma vez que o pH do mel pode ser influenciado pelo pH do néctar, composição do solo ou substâncias presentes na mandíbula das abelhas acrescidas durante o transporte do néctar até a colmeia. Valores de $\mathrm{pH}$ abaixo de 4,00 caracterizam um alimento muito ácido, o qual impede o crescimento microbiano, auxiliando na preservação do produto.

Pelas médias dos valores apresentados na Tabela 1 , observa-se que houve diferença significativa entre os valores de acidez $(\mathrm{P}<0,05)$, tendo o mel oriundo de Terra Roxa apresentado valor mais alto $\left(33,45 \pm 7,7 \mathrm{meq} \cdot \mathrm{kg}^{-1}\right)$ do que o de Santa Helena $\left(24,53 \pm 6,3\right.$ meq. $\left.\mathrm{kg}^{-1}\right)$, embora ambos se enquadrem no limite estabelecido pela legislação $\left(50\right.$ meq. $\left.\mathrm{kg}^{-1}\right)$, não caracterizando processo fermentativo, que pode ser explicado devido ao valor muito baixo de $\mathrm{pH}$. Segundo EvangelistaRodrigues et al. (2005), existe correlação entre os valores de $\mathrm{pH}$ e acidez, sendo que encontraram, para mel produzido na região do Cariri Paraibano, valores de 3,85 e $41,66 \mathrm{meq} \cdot \mathrm{kg}^{-1}$, ao passo que, naquele da região do Brejo Paraibano, os valores foram de 4,61 e 35,00 meq. $\mathrm{kg}^{-1}$, respectivamente, para cada parâmetro.

Os valores de acidez encontrados neste estudo, para o município de Santa Helena, foram semelhantes aos encontrados por Camargo (2011) para duas áreas produtoras de mel de

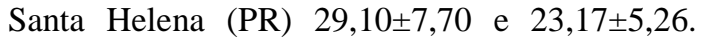
Alves et al. (2011), verificando a acidez em amostras de mel orgânico produzido nas ilhas do Rio Paraná, Estado do Paraná, encontraram valores entre 28,29 e 56,75, com média de $41,77 \pm 7,77 \mathrm{meq} \cdot \mathrm{kg}^{-1}$. Abadio-Finco et al. (2010)

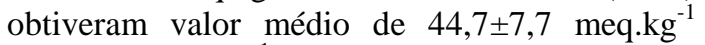
(35,0 a 59meq. $\left.\mathrm{kg}^{-1}\right)$, encontrando em $33,33 \%$ das amostras valores acima do permitido pela legislação vigente.

Tello Durán et al. (1996) comentam que a acidez do mel tem sua origem na variação dos ácidos orgânicos presentes em diferentes fontes de néctar (origem floral), pela ação da enzima glicose-oxidase, que origina o ácido glucônico pela ação das bactérias durante a maturação do mel e pelas quantidades de minerais presentes no mesmo. Segundo Marchini et al. (2004a), a acidez é um importante componente no mel, pois contribui para a sua estabilidade frente ao desenvolvimento de microrganismos.

A legislação brasileira de 1985 especificava para o parâmetro de condutividade elétrica a média de 200 a $800 \mu \mathrm{S} . \mathrm{cm}^{-1}$, mas a atual não estabelece valor mínimo e máximo permitido para tal parâmetro. Os valores médios de condutividade elétrica encontrados para o município de Terra Roxa são de $371,22 \pm 131,3 \mu \mathrm{S} . \mathrm{cm}^{-1}$ e de Santa Helena, 391,83 $\pm 50,5 \mu \mathrm{S} . \mathrm{cm}^{-1}$. Estes se encontram abaixo dos de Abadio-Finco et al. (2010), que encontraram valor de $585 \pm 203 \mu \mathrm{S} . \mathrm{cm}^{-1}$ para amostras de mel coletadas em municípios do sul do Estado de Tocantins; Alves et al. (2011) encontraram média de $1037,4 \pm 443,2 \mu \mathrm{S} . \mathrm{cm}^{-1}$ para mel orgânico produzido em ilhas do Rio Paraná, Estado do Paraná; e Moreti et al. (2009) verificaram valores que variaram de 154,7 a $1667,7 \mu \mathrm{S} . \mathrm{cm}^{-1}$ para amostras de mel coletadas no Estado do Ceará. 
Sodré et al. (2007) e Marchini et al. (2004a) mencionam que a condutividade elétrica é considerada um bom critério para a identificação da origem floral do mel, pois amostras com diferentes tipos florais têm valores diferentes de condutividade, e esse parâmetro é influenciado pelo conteúdo de cinzas, pH, acidez, entre outros. Souza et al. (2009) relatam que mel de mesma origem floral apresenta condutividade elétrica muito semelhante, apresar de origens geográficas e condições climáticas diferentes.

O valor médio para a análise de cinzas nas amostras de mel coletadas nos municípios de Terra Roxa e Santa Helena, no Paraná, foi de $0,19 \pm 0,1 \%$ para cada cidade, não diferindo estatisticamente entre si e encontrando-se de acordo com o valor estabelecido pela legislação vigente para tal característica no mel, que é de no máximo de $0,6 \%$. Esse valor está próximo aos encontrados pela maioria dos pesquisadores da literatura consultada, como Camargo (2011) que, estudando 54 amostras de mel coletadas no município de Santa Helena (PR), encontrou valores de cinzas de $0,17 \pm 0,05$ e $0,15 \pm 0,03$; Sodré et al. (2007), média de 0,18 $\pm 0,09 \%$ para 20 amostras de mel provenientes do Estado do Ceará; e Alves et al. (2011), valor médio de $0,17 \pm 0,08 \%$ em amostras de mel orgânico das ilhas do Rio Paraná. Os valores encontrados por Santos et al. (2011) foram superiores $(0,69 \pm 0,67 \%)$ para sete amostras de mel coletadas em estabelecimentos comerciais do município de Aracati-CE.

Segundo Marchini et al. (2005), a porcentagem de cinzas presentes no mel expressa a riqueza do material mineral; sendo que teores de cinza acima dos especificados sugerem adulteração por materiais inorgânicos, provenientes de objetos não especificados na descrição do mel, como terra, areia, pólen em excesso, etc.

A cor do mel está relacionada à condutividade elétrica e ao tipo de pólen encontrado no mel, sendo que, quando o mel for de cor mais clara, a condutividade será baixa. Nas amostras estudadas, os valores determinados para tal parâmetro foram baixos, $371,22 \mu \mathrm{S} . \mathrm{cm}^{-1}$ para Terra Roxa, sendo encontrada como cor predominante em $45 \%$ das amostras a cor âmbar, para o município de Santa Helena o valor médio de condutividade encontrado foi $391,83 \mu \mathrm{S} . \mathrm{cm}^{-1}$ e predominou a cor âmbar claro; tal fator pode estar relacionado ao tipo polínico das amostras de mel.

Camargo (2011), em análise de amostras de mel no município de Santa Helena (PR), encontrou predominância de cor âmbar claro em $42 \%$ das amostras, seguida por $26 \%$ de amostras classificadas como âmbar extra claro, seguida por $22 \%$ de cor âmbar e $10 \%$ com a coloração branca, encontrando forte relação com as cores apresentadas para este estudo.

No presente estudo, $30 \%$ das amostras de Terra Roxa e $25 \%$ das amostras de Santa Helena foram classificadas como âmbar escuro, escurecimento provavelmente devido ao tempo de armazenando, de aproximadamente um ano, ou ao tipo polínico apresentado pelas amostras.

Pesquisadores, como Sodré et al. (2007), encontraram, em 20 amostras de mel de $A$. mellifera coletadas em diferentes cidades do Estado do Ceará, predominância de cor âmbar claro $(40 \%)$, seguida por cor âmbar extra claro (25), âmbar (20), branco (10) e branco d'água (5). Moreti et al. (2009) encontraram predominância de cor branco d'água $(26,92)$, seguido por branco $(17,31)$, extra branco $(17,31)$, âmbar extra claro $(11,54)$, âmbar $(9,61)$, âmbar claro $(15,38)$ e âmbar escuro $(1,92)$ em 52 amostras de mel de A. mellifera coletadas em diferentes municípios do Estado do Ceará.

\section{CONCLUSÃO}

De acordo com as análises físico-químicas, as amostras de mel, de maneira geral, encontram-se dentro dos parâmetros recomendados pela legislação brasileira, com exceção do parâmetro umidade, que, em $37,5 \%$ das amostras, se apresentou acima de $20 \%$. Esses valores podem ser explicados pela colheita imatura do mel e foram repassados à Cooperativa de Apicultores regional, que presta assistência aos apicultores, para que estes sejam alertados e conscientizados quanto ao manejo correto. 


\section{REFERÊNCIAS}

ABADIO-FINCO, F.D.B.; MOURA. L.L.; SILVA, I.G. Propriedades físicas e químicas do mel de Apis mellifera L. Cienc. Tecnol. Aliment., v.30, p.706-712, 2010.

ALVES, E.M.; SEREIA, M.J.; TOLEDO, V.A.A. et al. Physicochemical characteristics of organic honey samples of Africanized honeybees from Parana River islands. Cienc. Tecnol. Aliment. v.31, p.635-639, 2011.

ASSOCIATION Of Official Analytical Chemistry - AOAC. Official methods of analysis of the Association of Official Analytical Chemists. Arlington: AOAC International, 1990. 500p.

BOLETIN Oficial Español (B.O.E). Orden de 12 de junio de 1986, de la Presidencia del Gobierno por la que se aprueban los métodos oficiales de analisis para la miel. B.O.E., n.145, 1986.

BRASIL. Ministério da Agricultura, Pecuária e Abastecimento. Instrução Normativa $\mathrm{n}^{\circ} 11$ de 20 de outubro de 2000. Aprova o Regulamento Técnico de Identidade e Qualidade do Mel. Diário Oficial da União, Brasília, DF, 23 de outubro de 2000.

CAMARGO, S.C. Aplicação de um Sistema de Informações Geográficas (SIG) no estudo da Apicultura na região oeste do Paraná. 2011. 72f. Dissertação (Mestrado em zootecnia) Universidade Estadual do Oeste do Paraná, Marechal Cândido Rondon.

CRANE, E. O livro do mel. 2.ed. São Paulo: Nobel, 1983. 223p.

EVANGELISTA-RODRIGUES, A.; SILVA, E.M.S.; BESERRA, E.M.F. et al. Análise físicoquímica dos méis das abelhas Apis mellifera e Melipona scutellaris produzidos em duas regiões no Estado da Paraíba. Cienc. Rural, v.35, p.1166-1171, 2005.

FUJII, I.A.; RODRIGUES, P.R.M.; FERREIRA, M. do N. et al. Caracterização físico química do mel de guaranazeiro (Paullinia cupana var. sorbilis) em Alta Floresta, Mato Grosso. Rev. Bras. Saúde Prod. Anim., v.10, p.645-653, 2009.
IBGE - INSTITUTO BRASILEIRO DE GEOGRAFIA E ESTATÍSTICA - 2010. Pesquisa da pecuária nacional. Disponível em: <http://www.sidra.ibge.gov.br/bda/tabela/listabl. asp? $=74 \& \mathrm{z}=\mathrm{p} \& \mathrm{o}=23>\quad$ Acessado em: 20/01/2012.

KÖPPEN, W. Climatologia. Com um estúdio de los climas de la tierra. México: FCE, 1948.478p.

MARCHINI, L.C.; MORETI, A.C. de C.C.; OTSUK, I.P. Análise de agrupamento, com base na composição físico-química de amostras de méis produzidos por Apis mellifera L. no Estado de São Paulo. Cienc. Tecnol Aliment., v.25, p.817, 2005.

MARCHINI, L.C.; SODRÉ, G.S.; MORETI, A.C.C.C. Mel brasileiro: composição e normas. Ribeirão Preto: A.S. Pinto, 2004a.111p.

MARCHINI, L.C.; SODRÉ, G.S.; MORETI, A.C.C.C. Composição físico-química de amostras de méis de Apis mellifera L. do Estado de Tocantins, Brasil. Bol. de Ind. Anim., v.61, p.1001-114, 2004b.

MORAES, R.M.; TEIXEIRA, E.W. Análise de mel (Manual técnico). Pindamonhangaba: Centro de Apicultura Tropical, 1998. 41p.

MORETI, A.C.C.C.; SODRE, G.S.; MARCHINI, L.C. et al. Características físicoquímicas de amostras de méis de Apis mellifera L. do estado do Ceará, Brasil. Cienc. Agrotec., v.33, p.191-199, 2009.

SANTOS, D.C.; OLIVEIRA, E.N.A.; MARTINS, J.N. Caracterização físico-química de méis comercializados no município de Aracati-CE. Acta Vet. Bras., v.5, p.158-162, 2011.

SILVA, C.L.; QUEIROZ, A.J.M.; FIGUEIRÊDO, R.M.F. Caracterização físicoquímica de méis produzidos no Estado do Piauí para diferentes floradas. Rev. Bras. Eng. Agric. Ambient., v.8, p.260-265, 2004.

SODRÉ, G.S.; MARCHINI, L.C.; MORETI, A.C.C.C. et al. Caracterização Físico-química de Amostras de Méis de Apis mellifera L. (Hymenoptera: Apidae) do Estado do Ceará. Cienc. Rural, v.37, p.1139-1144, 2007. 
SOUZA, B.A.; MARCHINI, L.C.; ODA-SOUZA, M. et al. Caracterização do mel produzido por espécies de Melipona Illiger, 1806 (APIDAE: MELIPONINI) da região nordeste do Brasil: 1. Características físico-químicas. Quim. Nova, v.32, p.303-308, 2009.

TELLO DURÁN, J.E.; CORTOPASSILAURINO, M.; ISSA, M.R.C. et al. Méis brasileiros: resultados de análises físico-químicas e palinológicas. In: $6^{\circ}$ CONGRESSO BRASILEIRO DE APICULTURA, 1996, Teresina. Anais... Teresina, 1996. p.403-429.
VIDAL, R.; FREGOSI, E.V. Mel: características, análises físico-químicas, adulterações $e$ transformações. Barretos: Instituto Tecnológico Científico "Roberto Rios", 1984. 95p.

ZENEBON, O.; PASCUET, N.S.; TIGLEA, P. Métodos físico-químicos para análise de alimentos. 4.ed. São Paulo: Instituto Adolfo Lutz, 2008. 1020p. 